\title{
True 3D kinematic analysis for slope instability assessment in the Siq of Petra (Jordan), from high resolution TLS
}

\author{
Giovanni Gigli (1), Claudio Margottini (2), Daniele Spizzichino (2), Heinz Ruther (3), and Nicola Casagli (1) \\ (1) Department of Earth Sciences, University of Firenze, Firenze, Italy, (2) ISPRA, Italian National Institute for \\ Environmental Protection and Research, Via Vitaliano Brancati 48, Rome 00144, Italy. claudio.margottini@isprambiente.it, \\ (3) Zamani Project, University of Cape Town (South Africa)
}

\begin{abstract}
Most classifications of mass movements in rock slopes use relatively simple, idealized geometries for the basal sliding surface, like planar sliding, wedge sliding, toppling or columnar failures. For small volumes, the real sliding surface can be often well described by such simple geometries. Extended and complex rock surfaces, however, can exhibit a large number of mass movements, also showing various kind of kinematisms. As a consequence, the real situation in large rock surfaces with a complicate geometry is generally very complex and a site depending analysis, such as fieldwork and compass, cannot be comprehensive of the real situation. Since the outstanding development of terrestrial laser scanner (TLS) in recent years, rock slopes can now be investigated and mapped through high resolution point clouds, reaching the resolution of few mm's and accuracy less than a cm in most advanced instruments, even from remote surveying. The availability of slope surface digital data can offer a unique chance to determine potential kinematisms in a wide distributed area for all the investigated geomorphological processes. More in detail the proposed method is based on the definition of least squares fitting planes on clusters of points extracted by moving a sampling cube on the point cloud. If the associated standard deviation is below a defined threshold, the cluster is considered valid. By applying geometric criteria it is possible to join all the clusters lying on the same surface; in this way discontinuity planes can be reconstructed, rock mass geometrical properties are calculated and, finally, potential kinematisms established. The Siq of Petra (Jordan), is a $1.2 \mathrm{~km}$ naturally formed gorge, with an irregular horizontal shape and a complex vertical slope, that represents the main entrance to Nabatean archaeological site. In the Siq, discontinuities of various type (bedding, joints, faults), mainly related to geomorphological evolution of the slope, lateral stress released, stratigraphic setting and tectonic activity can be recognized. As a consequence, rock-falls have been occurring, even recently, with unstable rock mass volumes ranging from $0.1 \mathrm{~m} 3$ up to over some hundreds $\mathrm{m} 3$. Slope instability, acceleration of crack deformation and consequent increasing of rock-fall hazard conditions, could threaten the safety of tourist as well as the integrity of the heritage. 3D surface model coming from Terrestrial Laser Scanner acquisitions was developed almost all over the site of Petra, including the Siq. Comprehensively, a point cloud of five billion points was generated making the site of Petra likely the largest scanned archaeological site in the word. As far as the Siq, the scanner was positioned on the path floor at intervals of not more than 10 meters from each station. The total number of scans in the Siq was 220 with an average point cloud interval of approximately $3 \mathrm{~cm}$. Subsequently, for the definition of the main rockfall source areas, a spatial kinematic analysis for the whole Siq has been performed, by using discontinuity orientation data extracted from the point cloud by means of the software Diana. Orientation, number of sets, spacing/frequency, persistence, block size and scale dependent roughness was obtained combining fieldwork and automatic analysis. This kind of analysis is able to establish where a particular instability mechanism is kinematically feasible, given the geometry of the slope, the orientation of discontinuities and shear strength of the rock. The final outcome of this project was a detail landslide kinematic index map, reporting main potential instability mechanisms for a given area. The kinematic index was finally calibrated for each instability mechanism (plane failure; wedge failure; block toppling; flexural toppling) surveyed in the site. The latter is including the collapse occurred in May 2015, likely not producing any victim, in a sector clearly identified by the susceptibility maps produced by the analysis.
\end{abstract}

